\title{
Erratum: Higher-order Fermi-liquid corrections for an Anderson impurity away from half filling: Nonequilibrium transport [Phys. Rev. B 97, 035435 (2018)]
}

\author{
Akira Oguri and A. C. Hewson \\ (Received 8 August 2018; published 27 August 2018)
}

DOI: 10.1103/PhysRevB.98.079905

We found another error in our paper after we had reported the first erratum [1]. In this second erratum, we briefly repeat the point that was reported in the previous one and then describe the additional corrections. All the errors occurred only in the applications to some special cases. The general Fermi-liquid relations for the self-energy, vertex functions, and transport coefficients, described in the original paper, are not affected by these revisions.

The first point is that in Figs. 4 and 5 of the original paper the results for the three-body correlation function for the up- and down-spins $\Theta_{\uparrow \downarrow}$, obtained at zero magnetic field, were plotted with the wrong sign. The revised figures are shown below [1].

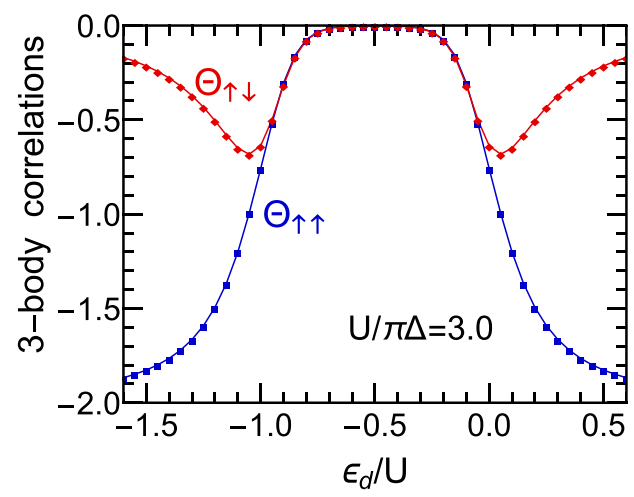

FIG. 4. Revised plot for $\Theta_{\uparrow \downarrow}$ with the correct sign in the lower panel of Fig. 4: Note that the plot for the parallel component $\Theta_{\uparrow \uparrow}$, which is also included, remains the same.

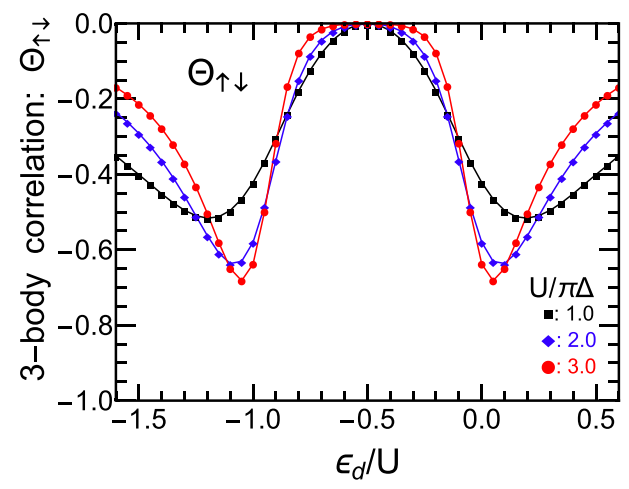

FIG. 5. Revised plot for the lower panel of Fig. 5: The antiparallel component $\Theta_{\uparrow \downarrow}$ is shown with the correct sign for $U / \pi \Delta=1.0,2.0$, and 3.0.
The statement about Fig. 5 given 11 lines below Eq. (5.22) should also be replaced by the following one: "The antiparallel component $\Theta_{\uparrow \downarrow}$ shows a minimum in the valence fluctuation region near $\epsilon_{d} / U \simeq-1.0$ and $0.0, \ldots$. as it has minima rather than maxima. These corrections do not affect the other zerofield results presented in Secs. V B and VI B.

The second error arose for the magnetotransport coefficients, which have been studied at half-filling $\epsilon_{d}=-U / 2$ in Secs. V C, VI C, and Appendix C. The central point is that there is an additional three-body term at finite magnetic fields, which was not taken into account properly in these sections. It is simply an error occurring in the application. The general Fermi-liquid relations that we described naturally yield this additional term. This term, which we write as $\Theta_{X}$, gives finite contributions together with the other three-body term $\Theta_{M}$ that has been described in detail in our paper. For this reason, Eq. (5.28) is replaced by the following form, adding the second line for $\Theta_{X}$ :

$$
\begin{aligned}
& \Theta_{M} \equiv-\frac{\sin \left(\pi m_{d}\right)}{2 \pi} \frac{1}{\chi_{\uparrow \uparrow}^{2}} \frac{\partial \chi_{\uparrow \downarrow}}{\partial h}, \\
& \Theta_{X} \equiv-\frac{\sin \left(\pi m_{d}\right)}{2 \pi} \frac{1}{\chi_{\uparrow \uparrow}^{2}} \frac{\partial}{\partial \epsilon_{d}}\left(\frac{\chi_{\uparrow \uparrow}-\chi_{\downarrow \downarrow}}{2}\right) .
\end{aligned}
$$

The following figure is a replacement for the lower panel of Fig. 8 in which $\Theta_{X}$ is also plotted as a function a magnetic-field $h$ at half filling together with $\Theta_{M}$. This term $\Theta_{X}$ is zero at $h=0$ and is very small for low-fields $h \lesssim T_{K}$ (inset).

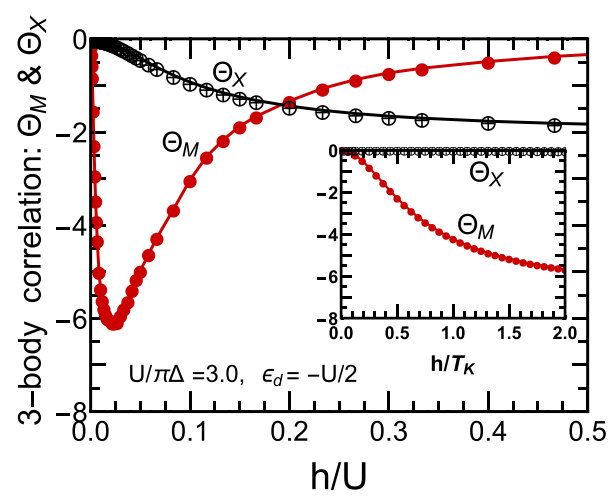

FIG. 8. Revised plot for the lower panel of Fig. 8: The result for $\Theta_{X}$ has been added. Here, $\Theta_{M}$ remains the same, and $T_{K}=0.02 \pi \Delta$ is defined at $h=0$ for $U=3 \pi \Delta$. 
However, it becomes comparable to $\Theta_{M}$ at $h \gtrsim 0.1 U$, and then it approaches $\Theta_{X} \rightarrow-2$ in the high-field limit $h \rightarrow \infty$ where the interaction $U$ becomes less important.
The contributions of $\Theta_{X}$ should also appear in Eqs. (5.23), (5.24), (5.25), (C5), (C6), (6.27), and (6.29): Equations (5.23) and (5.24) are replaced by

$$
\begin{aligned}
& \frac{1}{2} \sum_{\sigma} c_{T, \sigma} \stackrel{\xi_{d} \rightarrow 0}{\longrightarrow} \frac{\pi^{2}}{3}\left[\left(\chi_{\uparrow \uparrow}^{2}+2 \chi_{\uparrow \downarrow}^{2}\right) \cos \left(\pi m_{d}\right)-\frac{\sin \left(\pi m_{d}\right)}{2 \pi} \frac{\partial \chi_{\uparrow \downarrow}}{\partial h}-\frac{\sin \left(\pi m_{d}\right)}{2 \pi} \frac{\partial}{\partial \epsilon_{d}}\left(\frac{\chi_{\uparrow \uparrow}-\chi_{\downarrow \downarrow}}{2}\right)\right], \\
& \frac{1}{2} \sum_{\sigma} c_{V, \sigma} \stackrel{\xi_{d} \rightarrow 0}{\longrightarrow} \frac{\pi^{2}}{4}\left[\left(\chi_{\uparrow \uparrow}^{2}+5 \chi_{\uparrow \downarrow}^{2}\right) \cos \left(\pi m_{d}\right)-\frac{\sin \left(\pi m_{d}\right)}{\pi} \frac{\partial \chi_{\uparrow \downarrow}}{\partial h}-\frac{\sin \left(\pi m_{d}\right)}{2 \pi} \frac{\partial}{\partial \epsilon_{d}}\left(\frac{\chi_{\uparrow \uparrow}-\chi_{\downarrow \downarrow}}{2}\right)\right] .
\end{aligned}
$$

The statement below Eq. (5.24) should also be replaced by “The three-body term which enters through $\partial \chi_{\uparrow \downarrow} / \partial \epsilon_{d}$ has vanished because the contributions of $\uparrow$ and $\downarrow$ spin cancel each other out." Equation (5.25) is replaced by

$C_{T}^{h} \equiv \frac{\pi^{2}}{48}\left(W_{T}^{h}+\Theta_{M}+\Theta_{X}\right), \quad C_{V}^{h} \equiv \frac{\pi^{2}}{64}\left(W_{V}^{h}+2 \Theta_{M}+\Theta_{X}\right)$.

Equations (C5) and (C6) are replaced by

$$
\begin{aligned}
& \frac{\left(T^{*}\right)^{2}}{2} \sum_{\sigma} c_{T, \sigma}^{\mathrm{FMvMM}} \stackrel{\xi_{d} \rightarrow 0}{\longrightarrow} \frac{\pi^{2}}{48}\left(W_{T}^{h}+\Theta_{X}\right), \\
& \frac{\left(T^{*}\right)^{2}}{2} \sum_{\sigma} c_{V, \sigma}^{\mathrm{FMvDM}} \stackrel{\xi_{d} \rightarrow 0}{\longrightarrow} \frac{\pi^{2}}{64}\left(W_{V}^{h}-\Theta_{M}+\Theta_{X}\right) .
\end{aligned}
$$

Similarly, Eqs. (6.27) and (6.29) are replaced by

$$
\begin{aligned}
& C_{R, h}^{\mathrm{MA}} \equiv \frac{\pi^{2}}{48}\left(W_{R, h}^{\mathrm{MA}}+\Theta_{M}+\Theta_{X}\right), \\
& C_{\kappa, h}^{\mathrm{MA}} \equiv \frac{7 \pi^{2}}{80}\left(W_{\kappa, h}^{\mathrm{MA}}+\frac{13}{21} \Theta_{M}+\Theta_{X}\right) .
\end{aligned}
$$

Correspondingly, the upper panel of Fig. 9 should be replaced by the following figure. The contribution of $\Theta_{X}$ is important for high-fields $h \gtrsim 0.1 U$, and it makes the saturation level of the conductance coefficients deeper $\left(48 / \pi^{2}\right) C_{T}^{h} \rightarrow-3$

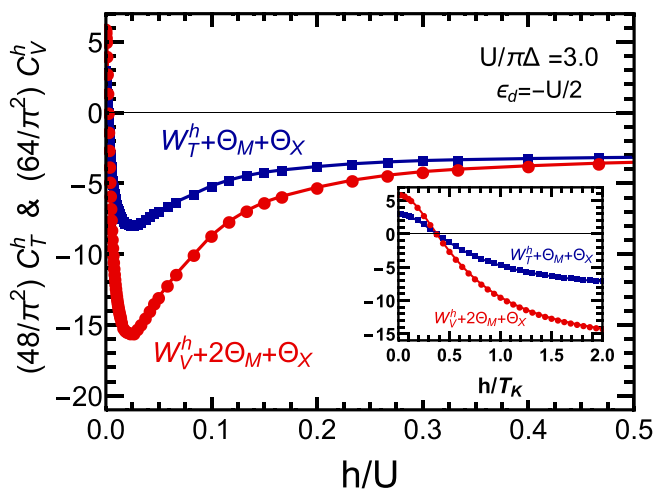

FIG. 9. Revised plot for the upper panel of Fig. 9: The coefficients $\left(48 / \pi^{2}\right) C_{T}^{h}=W_{T}^{h}+\Theta_{M}+\Theta_{X}$ and $\left(64 / \pi^{2}\right) C_{V}^{h}=W_{V}^{h}+$ $2 \Theta_{M}+\Theta_{X}$ are shown. and $\left(64 / \pi^{2}\right) C_{V}^{h} \rightarrow-3$ in the limit of $h \rightarrow \infty$. However, as $\Theta_{X}$ is very small at $h \lesssim T_{K}$, it does not cause visible changes for the low-field results of $C_{T}^{h}$ and $C_{V}^{h}$. For instance, there are no essential differences between the inset of the revised figure and that of the original one since the contribution of $\Theta_{X}$ becomes smaller than the size of the symbols on the lines for low-fields $0<h<2 T_{K}$. We have also confirmed that $\Theta_{X}$ causes no visible changes for the lower panel of Fig. 9 in which rescaled values of these two coefficients $\bar{C}_{T}^{h}$ and $\bar{C}_{V}^{h}$ are plotted for $0<h<1.5 T_{K}$.

The additional term $\Theta_{X}$ also contributes to the thermoelectric transport of dilute magnetic alloys, studied at half filling for finite magnetic fields. The lower panel of Fig. 12 should also be replaced by the following figure. Similar to the case of Fig. 9, major changes appear only at high magnetic-fields $h \gtrsim 0.1 U$. Correspondingly, the statements about Fig. 12, given six lines below Eq. (6.30), should also be replaced by the following one: "Therefore, at zero field, the coefficients are given by $\left(48 / \pi^{2}\right) C_{R, h}^{\mathrm{MA}}=W_{R, h}^{\mathrm{MA}}=3$ and $\left(80 / 7 \pi^{2}\right) C_{\kappa, h}^{\mathrm{MA}}=W_{\kappa, h}^{\mathrm{MA}}=13 / 7$ for large Coulomb interaction $U \gtrsim 2 \pi \Delta$ as $R_{W} \rightarrow 2$ and $m_{d}=0$. In the high-field limit $h \rightarrow \infty$, these two coefficients approach the following values $\left(48 / \pi^{2}\right) C_{R, h}^{\mathrm{MA}} \rightarrow 1$ and $\left(80 / 7 \pi^{2}\right) C_{\kappa, h}^{\mathrm{MA}} \rightarrow 1$, respectively, as $m_{d} \rightarrow 1, R_{W} \rightarrow 1, \Theta_{M} \rightarrow 0$, and $\Theta_{X} \rightarrow-2 . "$

As mentioned above, the main results of our paper are not affected by these revisions. Finally, we also note that Appendix

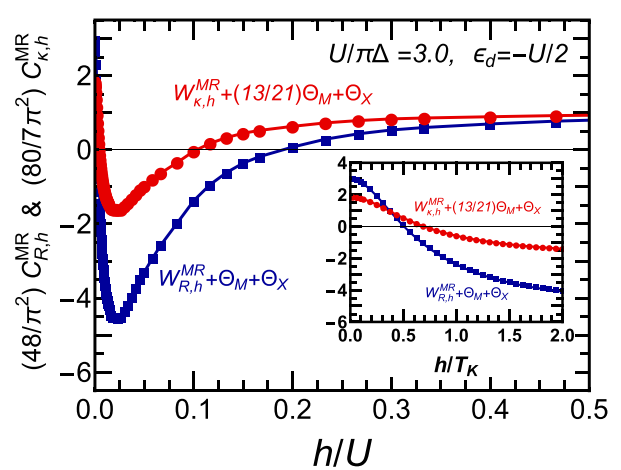

FIG. 12. Revised plot for the lower panel of Fig. 12: The coefficients $\left(48 / \pi^{2}\right) C_{R, h}^{\mathrm{MA}}=W_{R, h}^{\mathrm{MA}}+\Theta_{M}+\Theta_{X}$ and $\left(80 / 7 \pi^{2}\right) C_{\kappa, h}^{\mathrm{MA}}=$ $W_{\kappa, h}^{\mathrm{MA}}+(13 / 21) \Theta_{M}+\Theta_{X}$ are shown. 
$\mathrm{C}$ of our paper in which a comparison between the results of Filippone et al. [2] and ours was made, became no longer necessary as the revised paper of these authors has appeared very recently [3].

[1] A. Oguri and A. C. Hewson, Phys. Rev. B 97, 199902(E) (2018).

[2] M. Filippone, C. P. Moca, J. von Delft, and C. Mora, Phys. Rev. B 95, 165404 (2017).

[3] M. Filippone, C. P. Moca, A. Weichselbaum, J. von Delft, and C. Mora, Phys. Rev. B 98, 075404 (2018). 